This article has been retracted: please see INNOVATIONS in pharmacy retraction policy (https://pubs.lib.umn.edu/index.php/innovations/policies). This article has been retracted by the Editor and Publisher due to the inappropriate use of previously published work. 


\section{Framework for Patient Safety}

AK Mohiuddin, Assistant Professor

Department of Pharmacy, World University of Bangladesh, Bangladesh

\section{Abstract}

A commitment on quality objectives is a crucial element of quality policy in HROs, such as hospitals and other healthcare institutions. The quality of care includes objectives related to effectiveness, efficiency, and a patient's experience. Healthcare organizations are also aware of the importance of promoting safety practices and the resiliency analysis of the clinical practice in order to improve quality. Patient Safety Culture has been defined as the product of individual and group values, attitudes, competencies, and patterns of behavior that determines their commitment, style, and proficiency with the organization's health and safety programs. The safety culture of a health center offers an indirect means for its involvement in quality. Poor involvement of professionals in safety has negative consequences for patients. Envisioning the future of patient safety is more than an academic exercise. Appealing visions can help channel human energies, set new directions, and open the doors to alternative approaches. An outside observer is struck by three characteristics that are very different from the culture of the early 21st century: a deep sense of individual and institutional accountability for safety, an emphasis on fairness and transparency, and pervasive collabora tratar teamwork based on mutual respect. Speaking up is important for patient safety, but healthcare professionals often sitate to roice their concerns. Direct supervisors have an important role in influencing speaking up. However, good insight into t' re renship etween managers' behavior and employees' perceptions about whether speaking up is safe and worthwhile is still lad hg. The en areas in both instruments: strategy (inquiry on their commitment to the quality an rafet strategy, indicators' feedback, and risks maps), support systems for clinical decisions (digital record algorithms to mo ded ans an for accessibility to patient clinical

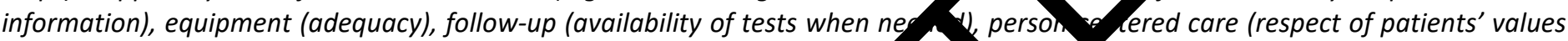
and preferences), evidence-based practice (practices in accordance with guiderines), elays (on scheduled tests, surgery, and outpatient care), and cost-effective treatments (adequacy).

Keywords: Medication; Errors; Risk, Reporting; Health care prafessiona. Safe+, Patient

Abbreviations: After Action Reviewers (AARs); Advers Oro Even (ADE); Potential Adverse Drug Event (PADE); Adverse Drug Reaction (ADR); Medical Error (ME); National Institute for He th an Care Ex ellence (NICE); People's Dispensary for Sick Animals (PDSA); Sound Alike Look Alike Drugs (SALAD); Computerized P ovis raeremry (CPOE); Drug-drug interactions (DDIs); Hospital Survey on Patient Safety Culture (HSOPS); Safety Attitudes estionna (SAQ); Agency for Healthcare Research and Quality (AHRQ); Situation, Background, Assessment, Recommendati (S) R); Institue for Health Care Improvement (IHI); Australian Pharmaceutical Advisory Council's (APAC); Medical Office Assi ant (MOA, Electronic Medical Record (EMR); Institute of Medicine (IOM); High-Reliability Organizations (HROs)

\section{Introduction}

Medicines are the commos merventions used in health care and safe use is im, rtant. Over the past 20 years, a number of initiatives aimed atmproving medication safety have been introduced into hospitals. The IOM estimated costs due to medical errors in the US of was approximately \$37.6 billion/year. About $\$ 17$ billion of it are associated with preventable errors. Clinicians, policymakers and patients now want to know whether progress has been made and where further improvement may be required. In 2016, medical errors were estimated to result in 250000 deaths annually, making

Corresponding author: AK Mohiuddin, Assistant Professor Department of Pharmacy

World University of Bangladesh, Bangladesh

151/8, Green Road, Dhanmondi, Dhaka - 1205, Bangladesh

Email: trymohi@gmail.com; Phone: +8801716477485 medical errors the third leading cause of death in the USA. Drug-related errors are the most common type of medical error and can result from discrepancies in patient medications during periods of transition of care. Around $60 \%$ of medication errors occur during patient admission, discharge or transfer of care. It is estimated that at least one medication error occurs each day for an average hospitalized patient. Err offered a similar conclusion relative to safety: flaws are unacceptable and common. The effective remedy is not to browbeat the health care workforce by asking them to try harder to give safe care, when in fact, the courage, hard work, and commitment of health care workers are the only real means to stem the tide of errors latent in the health care system. Growth in knowledge and technologies has never been so profound and prolific. However, research on the quality of care demonstrates that the health care system falls short in its ability to translate knowledge to practice and to apply new technologies safely and appropriately. These principles health care organizations could 
take now or as soon as possible to substantially improve patient safety include (1) providing leadership; (2) respecting human limits in process design; (3) promoting effective team functioning; (4) anticipating the unexpected; and (5) creating a learning environment.

\subsection{Important Definitions}

Active Error: Active errors are those taking place between a person and an aspect of a larger system at the point of contact. Active errors are made by people on the front line such as clinicians and nurses. For example, operating on the wrong eye or amputating the wrong leg are classic examples of an active error.

Adverse Event: Adverse events may be preventable when there is a failure to follow accepted practice at a system or individual level. An adverse event attributable to an error usually is a preventable adverse event.

Latent Error: These are errors in system or process design, faulty installation or maintenance of equipment, or ineffective organizational structure. These are present, but may go unnoticed for a long time with no ill effect.

Medical Error (ME): The failure to complete the intended plan of action or implementing the wrong plan to achieve an aim. An unintended act or one that fails to achieve the intende outcome.

Negligence: Failure to meet the reasonably expect stan ard of care of an average, qualified healthcare work a patient in question within similar circumstay s. Fon ample, the healthcare worker may not check $y$ the pat logy report which led to a missed cancer orythe surg n may have injured a nerve by mistaking it for a

Negligent Adverse Events: A sub egory preventable, adverse events that satisfy $t$ eleg crite ced in determining negligence. The injury cause hy surstandard medical management.

Near Miss: Any event that could have had an adverse patient consequence but did not. Near misses provide opportunities for developing preventive strategies and actions and should receive the same level of scrutiny as adverse events.

Noxious Episode: Untoward events, complications, and mishaps that result from acceptable diagnostic or therapeutic measures deliberately instituted. For example, sending a hemodynamically unstable trauma patient for prolonged imaging studies instead of the operating room. The result could be a traumatic arrest and death.

Patient Safety: The process of amelioration, avoidance, and prevention of adverse injuries or outcomes that arise as a result of the healthcare process [1].

\subsection{Scope of Safety Problems}

The provision of high-quality, affordable, health care services is an increasingly difficult challenge. Due to the complexities of health care services and systems, investigating and interpreting the use, costs, quality, accessibility, delivery, organization, financing, and outcomes of health care services is key to informing government officials, insurers, providers, consumers, and others making decisions about health-related issues. Appropriate medication use is a complex process involving multiple organizations and professions from various disciplines combined with a working knowledge of medications, access to accurate and complete patient information and integration of interrelated decisions over a period of time. The growing complexity of science and technology requires health care providers to know more more, monitor more, and involve more care provi ars than yer before. Current methods of organizing and delive ng care a not able to meet the new expectations of $\mathrm{p}$ cents a fan es because the knowledge, skills, care op ns, d fices, a medications have advanced more rapid th he care system's ability to deliver them saf $\psi$, effect ly ad efficiently. The potential for errors of or ssio or commission to creep into the process is extragdinary. Yorkflow analysis has often been used with the oal of improvimg efficiency. As primary care differs from hospital re in several important ways, e.g. in-patient aracter tics, organizational structure, relationships between hear are professionals and patients and types and outcomes atient-safety incidents, the risks associated with hospitalbased care should not be assumed to be the same as those associated with primary care. In response to financial pressure and incentives driving provider organizations, minimizing slack time has become important [2], [3].

\section{Understanding Error}

Clinicians' fears of lawsuits and their self-perceptions of incompetence could be dispelled by organizational cultures emphasizing safety rather than blame. To understand what is or is not known about medication related adverse events, common definitions must be established and understood. Organizations must come to a common understanding regarding MEs, reporting requirements, and risks to capture and act upon error potential within their own medication use systems. The potential benefits of intra-institutional and Webbased databases might assist pharmacists and other providers to prevent similar hazards and improve patient safety [4]. These definitions of $A D E, P A D E, A D R$ provide the following insights regarding adverse events and medication use:

- MEs are considered preventable while adverse drug reactions are generally are not.

- If an error occurs, but is intercepted by someone in the process, it might not result in an adverse event. These potential adverse events are often referred to as near misses.

- Capturing information regarding near misses could yield vital information regarding system performance. 


\section{Identifying Risk}

Two approaches to the problem of human fallibility are possible: the individual and the system approach. The individual approach focuses on the errors of individuals, blaming them for forgetfulness, carelessness or moral weakness. The system approach concentrates on the conditions under which individuals work and tries to build defenses to avert errors or mitigate their effects. Health care professionals are human and can make mistakes. Reporting an error is often viewed as professional failure or negligence and is followed by sanction or punishment of the individuals involved. Medications are inherently toxic, and there is a risk to taking them and, perhaps, not taking them. Each time a practitioner prescribes a product, a treatment risk versus benefit must be assessed. If a patient takes prescribed medications in a different manner than prescribed or if over-the-counter products and alternative agents are added, there are additional risks. Side effects and tragic rare reactions are also difficult to anticipate. This results in health care workers worrying constantly about the everpresent reality of error. Unfortunately, in many organizations, the response to error targets the people rather than the system involved in the production of an error [3], [5]. Reason has identified that there are a variety of defenses put into systems to provide the following functions:

- Create understanding or awareness of hazards

- Give guidance on how to operate safely

- Provide alarms and warnings when risk or danger is evident

- Place barriers between hazards and individuals or other systems

- Restore system to a safe state when conditions are not normal

- Contain or eliminated hazards if the barrier is not adequate

- Establish methods of escape and rescue should hazard containment fail

4. Targeting Medicath Safety The Microsystem Level Nelson and coll agues gge that understanding and nurturing clin al mi osyst so (Table 1) may create an opportunity for age to ard the goal of a safety and more effective alth cal sys $m$ [6].

Table 1. Scope of Ten Success Characteristics, derlying Principles, and Safety Impact

\begin{tabular}{|c|c|c|}
\hline Scope of Success Characteristic & Underlying Principle & Safety Impact \\
\hline $\begin{array}{l}\text { Leadership } \\
\text { - } \quad \text { Maintain constancy of purpose } \\
\text { - } \quad \text { Establish clear goals/expectations } \\
\text { - } \quad \text { Advocacy with in macro } \\
\text { - } \text { organization } \\
\text { - Formal, informal, on-the-spot }\end{array}$ & etting and eachind & $\begin{array}{l}\text { - } \text { Define safety vision } \\
\text { - } \quad \text { Identify constraints for safety } \\
\text { improvement } \\
\text { - } \quad \text { Allocate resources for plan development, } \\
\text { implementation, monitoring and } \\
\text { evaluation } \\
\text { - } \quad \text { Build input of microsystem to plan } \\
\text { - } \text { development } \\
\text { - } \quad \text { Plign quality and safety goals } \\
\quad \text { rovide update to Board of Trustees }\end{array}$ \\
\hline $\begin{array}{l}\text { Organizational support } \\
\text { - Recognition, resources, } \\
\text { - Enhance and legitimize } \\
\text { microsystem }\end{array}$ & & $\begin{array}{l}\text { - Work with clinical Microsystems to } \\
\text { identify patient safety issues and make } \\
\text { relevant local changes } \\
\text { - Put the necessary resources and tools into } \\
\text { the hands of individuals without making it } \\
\text { superficial }\end{array}$ \\
\hline $\begin{array}{l}\text { Staff focus } \\
\text { - Selective hiring } \\
\text { - } \text { Integration into culture and roles } \\
\text { competencies } \\
\text { - High expectations for performance, } \\
\text { continuing education, professional } \\
\text { growth, networking }\end{array}$ & $\begin{array}{l}\text { Human resource value chain that links } \\
\text { microsystem's vision with real people } \\
\text { for hiring, orienting, continuously } \\
\text { educating, retraining and providing } \\
\text { incentives }\end{array}$ & $\begin{array}{l}\text { - } \quad \text { Assess current safety culture } \\
\text { - } \quad \text { Identify gap between current culture and } \\
\text { - } \quad \text { safety vision } \\
\text { - } \quad \text { Plan cultural interventions } \\
\end{array}$ \\
\hline $\begin{array}{l}\text { Education and training } \\
\text { - Ongoing education } \\
\text { - } \quad \text { Organizational learning } \\
\text { - } \quad \text { aligned roles and competencies } \\
\text { Best use of people and resources }\end{array}$ & $\begin{array}{l}\text { Team approaches to training create } \\
\text { learning that is collaborative and focused } \\
\text { on quality, safety and integrated into } \\
\text { work flow }\end{array}$ & $\begin{array}{l}\text { Develop patient safety curriculum } \\
\text { - } \quad \text { Provide training and education of key } \\
\text { clinical and management leadership } \\
\text { Develop a core of people with patient } \\
\text { safety skills who can work across } \\
\text { microsystems as a resource }\end{array}$ \\
\hline
\end{tabular}




\begin{tabular}{|c|c|c|}
\hline $\begin{array}{l}\text { Interdependence of care team } \\
\text { - Trust } \\
\text { - } \quad \text { Collaboration } \\
\text { - } \quad \text { Willingness to help others } \\
\text { - } \quad \text { Appreciation of complimentary roles } \\
\text { - } \quad \text { Recognition of inputs to shared } \\
\quad \text { purpose }\end{array}$ & $\begin{array}{l}\text { Multidisciplinary team provides care and } \\
\text { every person is respected for individual } \\
\text { vital role }\end{array}$ & $\begin{array}{l}\text { - } \quad \text { Build PDSA into debriefings } \\
\text { - Use daily huddles for AARs and celebrate } \\
\text { identifying errors }\end{array}$ \\
\hline $\begin{array}{l}\text { Patient focus } \\
\text { - } \quad \text { Caring } \\
\text { - } \quad \text { Listening } \\
\text { - } \quad \text { Educating } \\
\text { - } \quad \text { Response to special requests } \\
\text { - } \quad \text { Innovating } \\
\text { - } \quad \text { Providing smooth service flow } \\
\text { - } \quad \text { Relationship with community } \\
\text { resources }\end{array}$ & $\begin{array}{l}\text { The patient is the common focal point, } \\
\text { it's why we're all here }\end{array}$ & $\begin{array}{l}\text { - } \quad \text { Establish patient and family partnerships } \\
\text { - } \quad \text { Support disclosure and truth about } \\
\text { medical error }\end{array}$ \\
\hline $\begin{array}{l}\text { Community and market focus } \\
\text { - } \quad \text { Partnership with community for } \\
\text { - } \quad \text { Outreach } \\
\text { - Innovation and excellence }\end{array}$ & $\begin{array}{l}\text { Resource exchange and information } \\
\text { sharing to assure that patient needs are } \\
\text { met }\end{array}$ & \\
\hline $\begin{array}{l}\text { Performance patterns } \\
\text { - } \quad \text { Patient outcomes } \\
\text { - } \quad \text { Cost avoidance } \\
\text { - } \quad \text { Streamlined delivery } \\
\text { - } \quad \text { Data feedback } \\
\text { - } \quad \text { Positive competition } \\
\text { - } \quad \text { Open dialog about performance }\end{array}$ & $\begin{array}{l}\text { Outcomes are routinely measur } \\
\text { feedback to Microsystems le } \\
\text { change based on data }\end{array}$ & $\begin{array}{l}\text {-evelop key safety measures } \\
\text { Create the "business case" for safety }\end{array}$ \\
\hline $\begin{array}{l}\text { Process improvement } \\
\text { - } \quad \text { Learning and redesign focus } \\
\text { - } \quad \text { Continuous care monitoring } \\
\text { - } \quad \text { Benchmarking } \\
\text { - } \quad \text { Tests of change } \\
\text { - } \quad \text { Staff empowered to innovate }\end{array}$ & work & $\begin{array}{l}\text { - Identify patient safety priorities based on } \\
\text { - } \quad \text { Address the work that will be required at } \\
\text { the microsystem level } \\
\text { - } \quad \text { Establish patient safety "demonstration } \\
\text { sites" } \\
\text { - Transfer the learning }\end{array}$ \\
\hline $\begin{array}{l}\text { Information and IT } \\
\text { - Information is key } \\
\text { - } \quad \text { Technology links infor } \\
\text { care } \\
\text { - } \quad \text { Communication and c }\end{array}$ & $\begin{array}{l}\text { or designed to } \\
\text { for the right }\end{array}$ & $\begin{array}{l}\text { - Enhance error reporting system } \\
\text { - Build safety concepts into information } \\
\text { flow (e.g. checklists, reminder systems, } \\
\text { etc) }\end{array}$ \\
\hline
\end{tabular}

\section{Collaboration Across the Medication Use Process}

Collaboration is essential to minimize patient risk in the medication use process. Health care providers within the organization need to understand and identify how these components function and who is involved in making these steps safe. Clear understanding of the critical safety issues at each one of these steps are of particular importance because the primary goal of adverse event identification is adverse event prevention. Each step can be considered a risk point and provides opportunities for internal checks and balances. At each step in the medication use process, it is often assumed physicians, nurses, pharmacists, and other health care providers in the organization play a role in patient evaluation
[7]. This evaluation would include assessing patient characteristics, medication selection, concurrent medications, medication dosage selection, and medication administration methods appropriate for the condition to be treated. The current system of prescribing, dispensing, administering, and monitoring, however, often places the responsibility on the individual to avoid making the mistake [8], [9]. Because this expectation seems unreasonable, organizations should focus efforts to improve medication use safety by using a systemsbased approach that identifies:

- Errors that occur most frequently

- Possible root causes of errors 
- Error prevention strategies to make it harder for the same or similar errors to occur

- If the organization has a system that makes it harder to commit an error, it will be more difficult for

\section{System Failures in the Medication Use Process}

Varieties of systems failures have been identified in hospitals that have studied factors associated with adverse events [3]. These system failures are listed below:

- Deficiencies in medication knowledge, including prescribing of incorrect medications, doses, forms, frequency, or routes of administration

- $\quad$ Failure to verify the identity or dose of medication administered, often due to look-alike packaging or similarities between medication names

- Inaccessibility of patient information including laboratory test results, current medications, and information on the patient's current condition

- Incorrect transcription of orders, often due to illegibility of the physician's handwriting

- $\quad$ Failure to note known medication allergies

- Inefficient order tracking, making it difficult to determine when a medication has been given, missed/discontinued or changed

- Poor communication between services, including between nurses and pharmacists

- Improper use of administration devices

- Lack of standardized dosing schedules or existing standards

- Lack of standardized system distribution

- Lack of standardized procedure

- Errors in preparation of (when performed in the

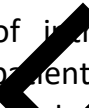
aross vits avenous edications

Poor information transfen patients are moved from one patient

- Inadequate or conexi ent syem for resolving conflicts related $n$ rcaumorders

- Deficiencies in staffin, or work assignments leading to excessive workloads, in onsistent availability of staff or inadequate management

- Lack of feedback and follow-up information on observed adverse drug events

\section{Classification of Medication Errors}

Medication errors can occur in:

- Choosing a medicine-irrational, inappropriate, and ineffective prescribing, under-prescribing and overprescribing;

- Writing the prescription-prescription errors, including illegibility;

- Manufacturing the formulation to be used-wrong strength, contaminants or adulterants, wrong or misleading packaging;

- Dispensing the formulation-wrong drug, wrong formulation, wrong label;

- Administering or taking the drug-wrong dose, wrong route, wrong freg wrong duration;

- Monitoring tb apy-to ing to alter therapy when required, errol sus alter jion.

The best way t ander and h medication errors happen and how to prem nonsider their classification, which can be ontextu dal, or psychological. Contextual classif atio deals wh the specific time, place, medicines, and peanle involv. Modal classification examines the ways in hich errors vccur (e.g. by omission, repetition, or substituti ). However, classification based on psychological eory is 0 be preferred, as it explains events rather than mencrescribing them. Its disadvantage is that it concentrates human rather than systems sources of errors. These classifications have been discussed in detail elsewhere [10], [11]. Mistakes can be divided into (i) knowledge-based errors and (ii) rule-based errors. Failures of skill can be divided into (iii) action-based errors ('slips', including technical errors) and (iv) memory-based errors ('lapses'). Knowledge-based errors can be related to any type of knowledge, general, specific, or expert. It is general knowledge that penicillin's can cause allergic reactions; knowing that your patient is allergic to penicillin is specific knowledge; knowing that co-fluampicil contains penicillins is expert knowledge. Ignorance of any of these facts could lead to a knowledge-based error. Rule-based errors can further be categorized as (a) the misapplication of a good rule or the failure to apply a good rule; and (b) the application of a bad rule. An action-based error is defined as 'the performance of an action that was not what was intended'. A slip of the pen, when a doctor intends to write diltiazem but writes diazepam, is an example. Technical errors form a subset of action-based errors. They have been defined as occurring when 'an outcome fails to occur or the wrong outcome is produced because the execution of an action was imperfect'. An example is the addition to an infusion bottle of the wrong amount of drug. Memory-based errors occur when something is forgotten; for example, giving penicillin, knowing the patient to be allergic, but forgetting [12-14]. 


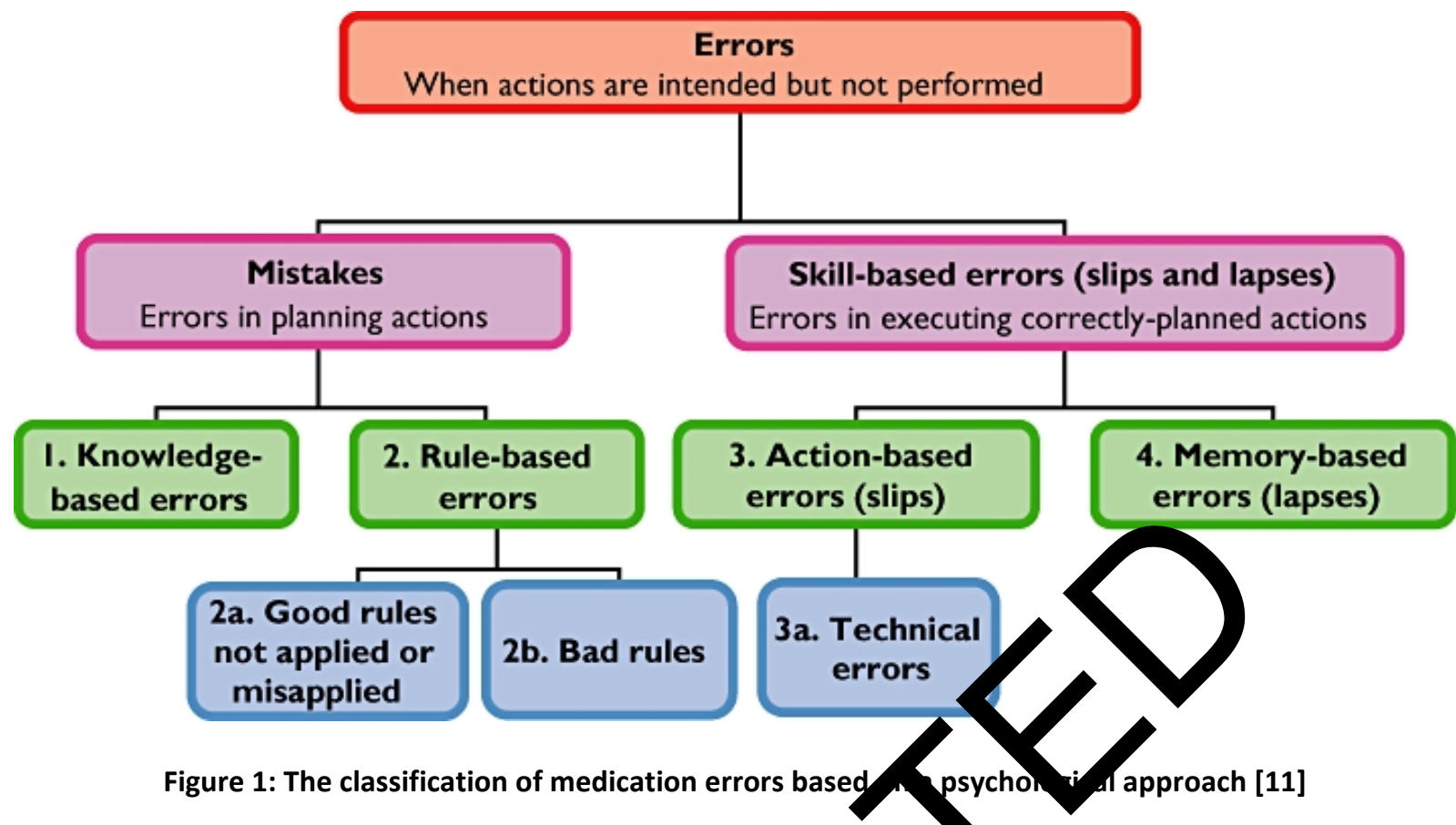

\section{Medication Error-Prevention Strategies}

To decrease the occurrence of medication errors, it is important to work toward developing strategies to combat the risk of medication errors at all steps in the medication-use process.

- Elimination of handwritten medical rec as nd physician orders/ Computerized providey order ttry systems

- Institute fail-safe tracking of edicatio and laboratory tests to ensure that panents reive conect medications and tests on tim

- Automated dispensing car

- Implement bar-coding

- Establish protocol standardized prar ces

- Provide all medio tion ackaging, ready for patient administ, fion

- Standardize medicatio grocedures such as protocols for the use of hazardous medications, medication terminology, and medication names

- Make it difficult for someone to do something wrong by error proofing

- Medication reconciliation

- Make relevant patient information available at the point of patient care

- CME

- Emergency-medicine clinical pharmacists

- Improve the patient's knowledge about treatment [3], [15]

\section{Recommendations for Prescribing Improvements}

he presd bing stage is one of the stages where medication ur most frequently, representing $71 \%$ of serious medication errors in hospital emergency. These errors occur Winth the physician orders the wrong drug, wrong dose, a drug to which a patient is allergic, or duplication in therapy. The nurse may then perpetuate the error by not questioning the physician. In addition, handwritten prescriptions can lead to catastrophic results, due to sloppy handwriting and confusion about decimal points. Many opportunities exist to improve the safety of the medication use process. The prescribing phase of the medication use process, however, encompasses the majority of medication errors that result in preventable ADEs. The most common drug group involved in MEs in Medicine ward was antibacterial followed by GI group, CVS group and IV fluids. This could be because a significant number of patients were hospitalized due to infectious diseases and CVS diseases. Presence of potential significant and serious DDIs in the prescriptions from Medicine and Pediatric ward. Potential significant DDIs were more common with CVS drugs such as atenolol, enalapril, digoxin, and furosemide. The risk of DDI was significant due to multiple drug therapy along with comorbidities in patients more than 40 years of age. While $\mathrm{GI}$ drugs such as ranitidine and ondansetron have been prescribed inappropriately, anticipating gastritis and vomiting in these patients. Prescribing of medications outside the accepted medical standards is known as an inappropriate prescribing. However, there were few irrational prescriptions (31\%) with unnecessary drugs such as ondansetron, ranitidine, multivitamins, folic acid, ceftriaxone, and metronidazole. The knowledge that ADEs can be prevented compels organizations to identify the factors or system failures that contribute to the 
errors in the prescribing phase. Such factors identified in the prescribing phase include:

- Availability of medication information at time of prescribing

- Access to patient information at time of prescribing

- Availability of dosing information at time of prescribing

- Availability of allergy information at time of prescribing

- Accuracy or completeness of order by prescriber

- Legibility of handwriting

- Use of abbreviations

- Use of decimals in expressions of weight and measure

- Use of varied units of measure

- $\quad$ SALAD [3], [17], [20]

\section{Error Potential in the Dispensing Phase}

The key player at this stage is the pharmacist. An example of the former type was a study in a UK hospital in which the researchers used semi-structured interviews of pharmacy staff about self-reported dispensing errors. The most common causes mentioned were: being busy (21\%), being short-staffed $(12 \%)$, being subject to time constraints $(11 \%)$, fatigue of healthcare providers (11\%), interruptions during dispensing $(9.4 \%)$, and SALAD (8.5\%). The dispensing process has both mechanical and judgmental components. This is alarming a infants ( $<1$ year) are vulnerable population for medicine AEs such as wrong dose, wrong route of administration $a_{\text {, }}$ of off-label drugs. In addition, pharmace netic or pharmacodynamic of drugs also differ in th atient population. A study in Saudi Arabia showe 47.3\% o rdose errors. IV fluid administration is involv $\mathrm{W}$ wrong ates worldwide. The reason for inaccurat dose adm istration is due to poor communication betwe heal'h care professional team, missed labeling of IV fla id improper use of instruments. As a result, pre ron disp ing errors will require a comprehensive proag includ sovaluation of:

- Work environment: Orkload, distractions, physical location of service, hours foperation

- Inventory management: outdated or unused products, look-alikes, sound- alike, clutter, labeling, purchasing of unit of use products

- Information resources: available references, updates, consultants, computer or decision support technology

- Performance evaluation: evaluation of staff competency and practice skill, knowledge and behaviors, crosschecking redundancies

- $\quad$ Patient involvement: patient education and review with show and tell techniques
Several critical steps have been advocated for improving dispensing accuracy:

- $\quad$ Secure or sequester high-risk medications

- Develop and implement standardized storage procedures

- $\quad$ Reduce distraction potential and improve workflow in dispensing environment

- Use reminders (labels, computer alerts) to prevent SALAD mix-ups

- Keep prescription order, label, medication and the medication container together throughout dispensing process

- Perform a final check on prescription content including veri arom with original prescription order and

- Enter a man ycturer entification code into the comp er profilo nd in prescription label

- Per rm a rnal chack on the prescription label, if ossio using domation such as bar-coding

Provide it counseling [16], [17], [20]

\section{2ror Poted ial in the Transcribing Phase}

ntering incorrect data onto the medication administration ecord by onmedical personnel can be a factor. Transcription ors or ar when a communication issue occurs between the orescriber and the individual dispensing or administering the mudication. These can develop from handwriting errors, but can also be secondary to unclear or misinterpreted orders. The uniqueness of transcribing errors lies in the fact that they are due to communication failures rather than knowledge opportunities; they are $100 \%$ preventable. The use of CPOE systems can essentially eliminate the occurrence of these errors by having providers enter medication orders directly into the system. Some contributing factors to these errors include the multitude of names, dosage forms, and strengths of various agents. Soundalike medications and abbreviations also provide a significant obstacle to eliminating these errors as well [15], [17].

\section{Error Potential in the Administration Phase}

The act of physically administering the drug to the patient represents the very last moment providers can catch errors before they can do harm. Nurses are absolutely critical personnel at this stage. These errors typically arise when the wrong drug is administered, or the right drug is administered in the wrong dose, via the wrong route, or with an incompatible co-administered drug. These errors encompass the wrong drug, the wrong patient, and the wrong route. A common error in hospitals involves patient-controlled analgesia pumps. Although the advantage of the pump is the ability of the patient to obtain the right dose, if the pump has not been programmed correctly the patient may risk serious side effects. Busy schedule, urge to complete work as early as possible, and missing double check/cross checking of prescription orders can 
lead to wrong route of administration. These reasons not only increase the patient risk, but also increase the cost of treatment. The administration phase, serves as a last final check on processing the entire medication order itself and includes:

- Evaluating the written order for appropriateness and completeness

- Assuring appropriate indication for use

- Evaluating and interpreting use of terminology and order method (abbreviation, units of measure, use of verbal orders)

- Dosing calculation or verification

- Identification of the patient

- Timing of treatment in context of other therapies

- Preparation and possibly dispensing of medication

- Proper use of medication devices

- Patient education

- Documentation of treatment [3], [15], [17], [20]

\section{Errors during patient discharge and monitoring}

Medication errors are a common and significant problem, particularly when patients transition between healthcare providers. Discrepancies are especially prevalent on hospital admission and discharge. As patients move between health providers and settings, discrepancies and miscommunication in clinical records are common and lead to serious medication errors. Hospital admissions and discharges, interdepartment transfers, or care shared between a specialist and a GP, are often dangerous times for patients, especially those term conditions or taking multiple medicines. Th patients with one or more medication discrepa discharge has been reported at $14 \%$ to different research. Of the errors discharge in a prospective observation had the potential to result in Recommendations to avoid medi
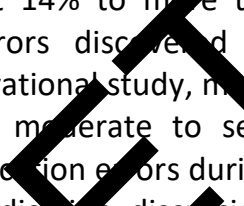
th that $30 \%$ in at ho yital patient discharge include a medico disc osion with the patient featuring open-end $\mathrm{qu}$ stion active listening to effectively share infor ation atient education focused on medications such as insulin ministration, and providing the patient with an accurate list 0 medications to be taken after discharge. The monitoring stage involves the activities following administration of a medication and observing the impact of pharmacotherapy. This is not only within the traditional sense of monitoring on the inpatient side but also includes patients discharged to home and ensuring that appropriate follow-up occurs. Poor medication management during or immediately after hospital admission increased the risk of readmission in the next month by $28 \%$. The key to reducing serious medication errors and patient harm is to ensure timely, accurate handover of medicines at all transition points in care. Smooth transitions require competent and coordinated responses from health professionals focused on the individual's needs. Implementing medication review by a pharmacist at hospital admission and creating a discharge medication reconciliation form and report that patients can take home with them reduced medication errors from nearly $60 \%$ to $30 \%$. More than half of Canadians (55\%) and Americans are estimated to have less than adequate health literacy skills. It is important that patients are given adequate discharge instructions and are counseled on potential self-monitoring techniques, as well as any possible harmful side effects that may occur [20-25].

\section{Changing Systems Within Organizations}

Within the United Kingdom of Great Britain and Northern Ireland, for example, there are about 100000 reports of patient-safety incidents from England and Wales every month. Real and potential ME in inpatients can be identified mainly reviewing drug prescriptions by the pharmacists. The most of interventions are focused in wrong anti-infective dosages. Prevention strategies for from analyses of the reports are contributi direc to patient safety. Health institutions, which are nown to pe complex organizations, have over the yea devise mpr ement strategies and added quality to the nealth care vice. Patient's safety culture reflects the pel ans 9 processes, norms, and attitudes relating a cultu. of reventable errors shared by health profe rono in the a.ivery of care. In health environments, behaviors and titudes shape the culture of each organization. ne sharing of veliefs, values, and attitudes related to the batient's safety culture influences the outcome and ganizat nal aspects. Quality in hospital services means pron the patient with multidisciplinary care at minimal risk. - imefore, implementing improvements in organizational and safety culture enhances quality. Among the validated tools, the HSOPS and the SAQ, both created in the USA, are widely cited in research that aims to assess the safety culture of patients in different countries. The culture of safety is measured by the staff perspective. For each dimension, percentages above $75 \%$ are considered as strengths and below $50 \%$ are areas that need improvement. The following items have routinely been identified as a top 10 list for improvement in general:

- Improving knowledge about medications (availability, access and timeliness)

- Dose/identity tracking of medications (process understanding of distribution)

- Available patient information (availability, access, accuracy and timeliness)

- Order transcription (elimination of process)

- Allergy defense (hard stop capabilities, access to patient information)

- Medication order tracking (streamlining and effective communication of patient needs)

- Communication (patient information, system performance, medication use)

- Device use (standardization and competency regarding use)

- Standardization of medication dose

- Standardization of medication distribution [3], [19], [26, 27] 


\begin{tabular}{|l|l|}
\hline \multicolumn{3}{|c|}{ Exhibit 1. WHO's International Classification for Patient Safety: descriptions of harm severity [28] } \\
\hline None & Outcome was not symptomatic or no symptoms were detected and no treatment was required. \\
\hline Mild & $\begin{array}{l}\text { Patient outcome was symptomatic, symptoms were mild, loss of function or harm was either minimal } \\
\text { or intermediate but short-term and no intervention or only a minimal intervention, e.g. extra } \\
\text { observation, investigation, review or minor treatment, was required. }\end{array}$ \\
\hline Moderate & $\begin{array}{l}\text { Patient outcome was symptomatic, required more than a minimal intervention, e.g. additional } \\
\text { operative procedure or additional therapeutic treatment, and/or an increased length of stay and/or } \\
\text { caused permanent or long-term harm or loss of function. }\end{array}$ \\
\hline Severe & $\begin{array}{l}\text { Patient outcome was symptomatic, required a life-saving or other major medical/surgical } \\
\text { intervention, shortened life expectancy and/or caused major permanent or long-term harm or loss of } \\
\text { function. }\end{array}$ \\
\hline Death & On balance of probabilities, death was caused or brought forward in the short-term by the incident. \\
\hline
\end{tabular}

\section{Conduction of a Root Cause Analysis}

DDIs account $6-30 \%$ of all adverse drug events and can increase occurrences of ME. Furthermore, self-medication, poor communications between the prescriber and the patient, and even demand of the patient for medicine for each symptom, unethical drug promotion and inducements increases irrational prescribing. Medication-error reporting is an essential aspect of limiting medication-error occurrence and the development of medication error-prevention strategies. Despite the profound impact that these errors have on the system, they largely go underreported, mainly due to the fear of repercussions Although challenging, medication errors should be viewed a valuable learning exercises to improve care and limit their recurrence, and hospital management should avg punitive action against individuals involved errors, as this discourages reporting and minn opportunities. Every medication error that oes un vorted represents an error that will continue be eplicated and continue to put patients at risk. Errors lat are re sted allow for the development of potential untermeasures to either eliminate them altogether or mits te trir impact. The large majority of errors are the resuld de th thexist within the underlying systems of $p$ ctice athe an within human practitioners, as they mer v se vessel by which these shortcomings are manife d. One population that is particularly vulnerable are pat ts admitted to the ICU. ICU patients have a combination of rapidly changing medical conditions, laboratory values, and medications, which present a particular challenge for clinicians in practice in every aspect of patient care. Medication errors can occur in different phases (prescribing, distribution, administration, and monitoring) of the medication process and have a significant impact on morbidity and mortality. Medication error analysis requires a structured approach including: detection, reporting, and analysis, in order to provide the most efficient and practical information to the ICU team. In addition, a particular focus is made on the implementation of medication error prevention strategies such as evidenced-based protocols, team education, and technology. In an effort to reduce medication error rates in the ICU requires a coll orative, ulti-disciplinary approach in order to be effectivgand nsisten through time. Although the elimination of a dication ro should be the goal of any organization, $\mathrm{simp}$ tant tovecognize that the occurrence of medication aro an ine cable part of clinical practice. There are sey key to su for health care organizations to consiarer as conduct a root cause analysis:

- Identify a multidisciplinary team to assess the error, f lure, or adverse event of interest tablish a way to communicate findings and data elements required to conduct the analysis

Create a plan with target dates, responsibilities, and measurement/data collection strategies required for the investigation

- Define all elements of the process and issues clearly

- Brainstorm all possible causes or potential causes

- Identify interrelationships of causes or potential causes

- Sort, analyze and prioritize cause list

- Determine which processes and systems are part of the investigation

- Determine special and common causes

- Begin the design and implementation of the change while engaging in the root cause analysis

- Repeat each of the steps listed previously as appropriate

- Focus on being thorough (Ask why) and credible (Be consistent, dig deep, and leave no stone unturned!)

- Target system improvement, particularly the larger systems

- Redesign to eliminate root cause(s)

- Measure and assess new design [3], [15], [18] 
Exhibit 2. Primary Care Harm Severity Classification System [27]

\begin{tabular}{|c|c|c|}
\hline Severity & Definition & Examples \\
\hline No harm & $\begin{array}{l}\text { Any incident that ran to completion but no harm occurred to } \\
\text { the patient }\end{array}$ & $\begin{array}{l}\text { Patient received azathioprine but missed routine } \\
\text { hematological monitoring for several months. No } \\
\text { harm incurred }\end{array}$ \\
\hline $\begin{array}{l}\text { No harm } \\
\text { outcome } \\
\text { due to } \\
\text { mitigating } \\
\text { action }\end{array}$ & $\begin{array}{l}\text { Any incident that had the potential to cause harm to a patient } \\
\text { but resulted in no harm }\end{array}$ & $\begin{array}{l}\text { A receptionist issued an incorrect prescription that } \\
\text { indicated a patient should take one tablet twice daily } \\
\text { instead of once daily. The chemist providing the } \\
\text { tablets, who had dispensed to the patient previously, } \\
\text { noted the error and corrected the regimen }\end{array}$ \\
\hline Mild harm & $\begin{array}{l}\text { Incident in which: (i) patient was harmed, with mild and short- } \\
\text { term impact, on physical, mental or social functioning, that } \\
\text { was expected to resolve in a few hours; (ii) patient was } \\
\text { harmed but required no or minimal intervention/treatment, } \\
\text { e.g. anti-emetic, oral antibiotic or repeat of a minor procedure } \\
\text { such as vaccination or insertion of contraceptive implant; } \\
\text { and/or (iii) patient or their loved ones experienced transient } \\
\text { emotional distress but no long-term consequences and } \\
\text { incident report contains words, e.g. angry, anxious, confused, } \\
\text { distressed, frightened, frustrated, humiliated or upset, that } \\
\text { might describe a feeling that occurs at the time of the incident } \\
\text { but soon passes }\end{array}$ & $\begin{array}{l}\text { An on-call primary-care physician prescribed oral } \\
\text { analgesic for a patient who could not swallow. A } \\
\text { second physician also made a prescription error, } \\
\text { leaving patient in pain for three hours. }\end{array}$ \\
\hline $\begin{array}{l}\text { Moderate } \\
\text { harm }\end{array}$ & 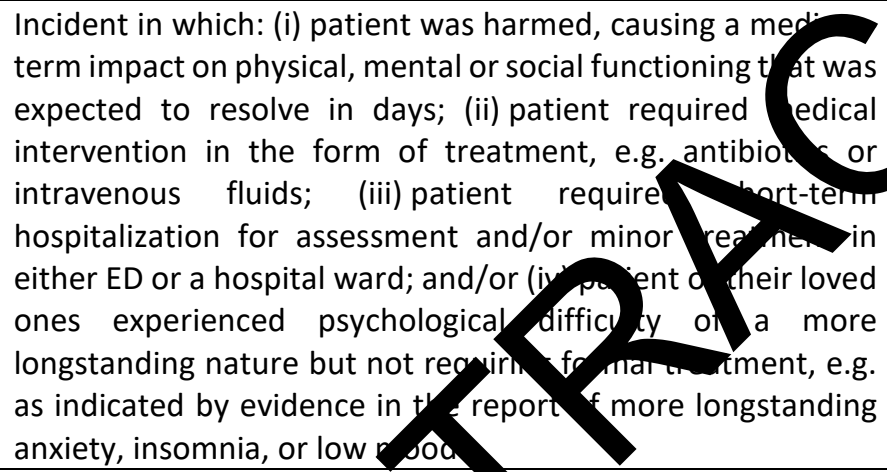 & $\begin{array}{l}\text { A pant was prescribed amoxicillin despite being } \\
\text { known to have penicillin allergy. Although the error } \\
\text { as corrected and the patient given clarithromycin, } \\
\text { he patient claimed to have lost trust in doctors and } \\
\text { to be extremely anxious about taking the } \\
\text { clarithromycin }\end{array}$ \\
\hline $\begin{array}{l}\text { Severe } \\
\text { harm }\end{array}$ & 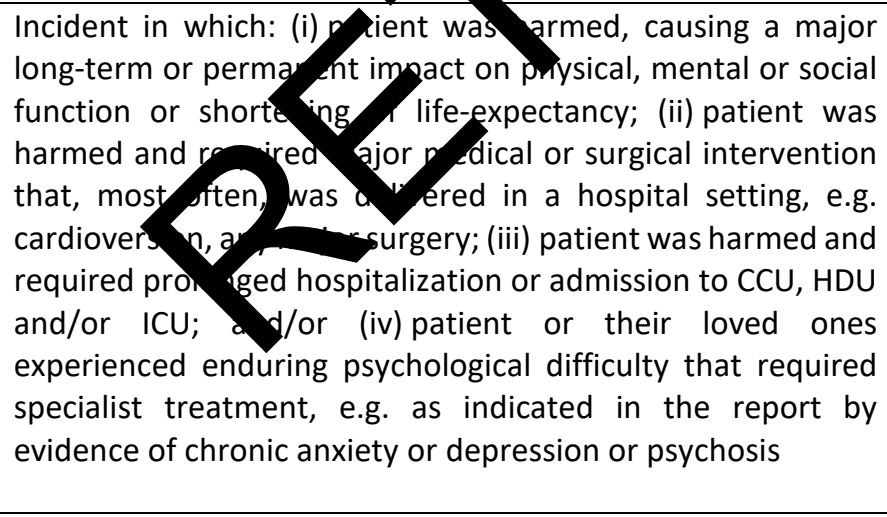 & $\begin{array}{l}\text { An epileptic child who had been prescribed } \\
\text { phenobarbital was admitted with symptoms of } \\
\text { drowsiness and had decreased tone for three days. } \\
\text { He was ventilated and immediately transferred to } \\
\text { the ITU because he had a low GCS score. His blood } \\
\text { concentration of phenobarbital was found to be } \\
\text { abnormally high. When the patient's own supply of } \\
\text { phenobarbital was checked, the original } \\
\text { manufacturer's label gave the strength as } 25 \mathrm{mg} / \mathrm{mL} \\
\text { but the erroneous community pharmacy's label } \\
\text { indicated } 25 \mathrm{mg} / 5 \mathrm{~mL} \text {. The child had been receiving } \\
\text { five times the prescribed dose }\end{array}$ \\
\hline Death & $\begin{array}{l}\text { Incident in which, on the balance of probabilities, death of the } \\
\text { patient was caused or brought forward in the short term by } \\
\text { the incident }\end{array}$ & $\begin{array}{l}\text { A patient contacted an out-of-hours service by } \\
\text { telephone, reporting feeling unwell, vomiting and a } \\
\text { rash on his stomach. A physician, who returned the } \\
\text { patient's call, diagnosed a viral illness and asked the } \\
\text { patient to make arrangements for a relative to collect } \\
\text { a prescription for an anti-emetic. Within } 90 \text { minutes, } \\
\text { however, the patient had deteriorated and been } \\
\text { brought to the ED of his hospital. The patient was } \\
\text { diagnosed with meningococcal septicemia and died }\end{array}$ \\
\hline
\end{tabular}


Insufficient $\quad$ Incident for which the report carries insufficient information detail to evaluate the severity of harm. The report may describe an error or outcome that was not the result of primary health care, e.g. a fall in the waiting room. Alternatively, it may fail to describe any outcome or it may describe a patient-safety incident but give insufficient information to classify the severity of harm of the outcome, e.g. it may record a delay in getting an appointment but not describe the consequences of the delay for the patient
A patient provided samples for histology and cytology, but the provider collecting the samples in specimen pots forgot to label the pots

* ED: emergency department; CCU: coronary care unit; GCS: Glasgow coma scale; HDU: high dependency unit; ICU: intensive care unit; ITU: intensive therapy unit.

16. Sources of Learning About Patient Safety

- The Agency for Healthcare Research and Quality (AHRQ)

- The American Hospital Association (AHA)

- Anesthesia Patient Safety Foundation (APSF)

- Annenberg Patient Safety Conferences

- Institute for Healthcare Improvement (IHI)

- Institute for Safe Medication Practices (ISMP)

- Joint Commission on Accreditation of

- Healthcare Organizations (Joint Commission, JCAHO)

- Leapfrog Group

- Malcolm Baldrige National Quality Program

- Massachusetts Coalition for the Prevention Medical Errors

- Minnesota Hospital and Healthcare (MHHP)

- National Academy for State Health

- National Coalition on Health Car

- National Committee for Qual A urance (N QA)

- National Patient Safety Fo ndation (PSF)

- National Quality Forum ,NQF)

- United States Pharn cope Convention (USP) [29]

17. Role of Patients in $M$ dicat Lereors

This area is relatively und researched and there remain several unanswered question. Little is known about how patients understand drug related problems or how they make attributions of adverse effects. Some research suggests that patients' cognitive models of adverse drug reactions bear a close relationship to models of illness perception. Recent NICE guidelines recommend that professionals should ask patients if they have any concerns about their medicines, and this approach is likely to yield information conducive to the identification of medication errors [30].
18. Developing a medication communication framework Communication breakdow "laboration failure, and inability to recognize the clinica deterior on of patients are the main reasons for the occurro se of se pus events in the hospital setting. Sharing $p$ cent-spo ific $b$ alth care information during handoff requip situr onal a areness. In the hospital setting, most of tb co "anicati related to patient care occurs between urses, $A$ ro cists and physicians. Challenges of comn rnica on amons health care providers are not limited to diffunces in training and reporting expectations. The stimated rate of preventable ADEs, caused by medication errors, is 5 million per year in the US. There is a link between edicatid errors and medication communication; colmmernication between providers and between providers and ants, within and across care settings, have been identified as sources of medication error. The Joint Commission, AHRQ, $\mathrm{IHI}$, and WHO recognize SBAR as an effective communication tool for patients' handoff. SBAR is a reliable and validated communication tool which has shown a reduction in adverse events in a hospital setting, improvement in communication among health care providers, and promotion of patient safety. Despite its importance, communication is often an embedded component (not the focus) of models that explore medication management workflow, including describing: medicine pathways; e-prescribing; and patient safety [31,32].

Exhibit 3. Components for achieving continuity in medication management across the continuum of care outlined by APAC [33].

- Decision to prescribe medicine

- Record of medicine order/prescription

- Review of medicine order/prescription

- Issue of medicine

- Provision of medicine information

- Distribution and storage

- Administration of medicine

- Monitor for response

- Transfer of verified information 
Medication management requires extensive collaboration and communication across roles and care settings, which can reduce (or contribute to) medication-related errors. Medication management involves key recurrent activities: determine need; prescribe; dispense; administer; and monitor/evaluate with information communicated within and between each activity; medication communication is therefore embedded within the framework. Coordinating activities include: relaying messages between care providers and relaying messages between patients and care providers; requesting or transmitting patient information for current or historical medication records; requesting and confirming appointments and referrals; and requesting, confirming, or alerting roles of the status of medication coverage. While the content of what these roles communicate falls within other medication communication activities (e.g., prescribing, dispensing, monitoring), their responsibility is to facilitate the coordination of this information between roles (e.g., between a family physician and a pharmacist) [34].

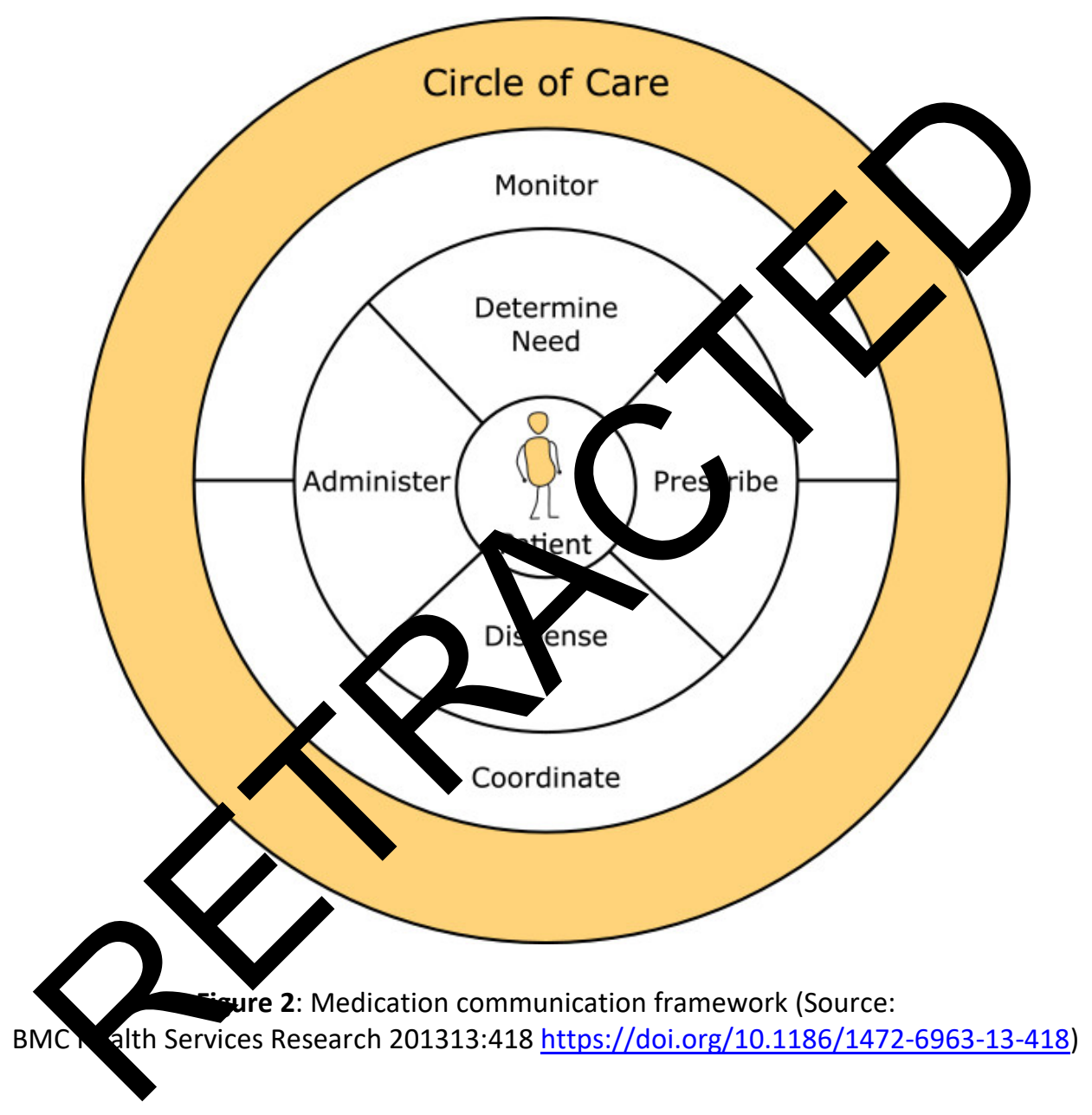

While the five medication management categories (determine need, prescribe, dispense, administer, monitor/evaluate) had alignment with communication activities, not all communication activities could be mapped directly to the medication management framework. The medication communication activities are mapped along pathways between roles. Interestingly, fewer coordinate communication pathways observed in the integrated clinic versus the non-integrated clinics. In a non-integrated clinic, the pharmacy is off-site and coordination is regularly required to link the family physician and pharmacist together to confirm, for instance, current medications as reflected in the electronic provincial medication repository or to discuss a dispensing alert (e.g., for a contraindicated medication). In the integrated clinic with a pharmacy on-site, however, conversations between the pharmacist and family physician generally occur directly in the pharmacy or clinic hallway [34]. 
Exhibit 4. Important definitions regarding Medication communication [33]

- Determine need involves communication around activities to determine the need for medication, e.g., a doctor and patient discussing the patient's complaint;

- Prescribe focuses on communication around prescribing activities, e.g., a patient and doctor discussing the details of a new prescription;

- Dispense focuses on communication around dispensing activities, e.g., a pharmacist and physician resolving an alert for a duplicate medication;

- Administer involves communication around medication administration activities, e.g., a pharmacist and patient discussing medication administration instructions;

- Monitor/Evaluate focuses on communication around medication monitoring and evaluation, e.g., a patient and Home and Community Care nurse discussing medication compliance. The monitor/evaluate activity was often used to inform the other medication communication activities for medication decision-making; and

- Coordinate communication focuses on the coordination of medication information between roles, e.g., a MOA transmitting a request for information between a pharmacist and a family physi

Currently, the "gold standard" of obtaining the best possible medication history involves a pharmacist compiling patient history, obtaining patient and office medication lists, pharmacy data, and discharge summaries upon admission. Approaching discharge, the pharmacist reviews changes in medications, describes indications and reviews discontinued medications. Post discharge, the pharmacist contacts the patient again to resolve any unanswered questions. Many institutions are not yet current with these best practices or have limited pharmacy staff to complete such tasks. The process of medication

reconciliation is then agated $t$ nurses or physicians who often do not hav /me or our s to ensure a high-degree of accuracy. Alth ugh ts wolnow is effective for a single hospitalizat n, a labo us task that must be repeated at every tr sition o ar and does not address the issue of creating in tiple our-of-date medication lists. Medication rem iliation a Joint Commission National Patient Safety oal and a core measure of Stage 2 meaningful use. However, cospitals nd EMR vendors have struggled to meet this andate 33-37].

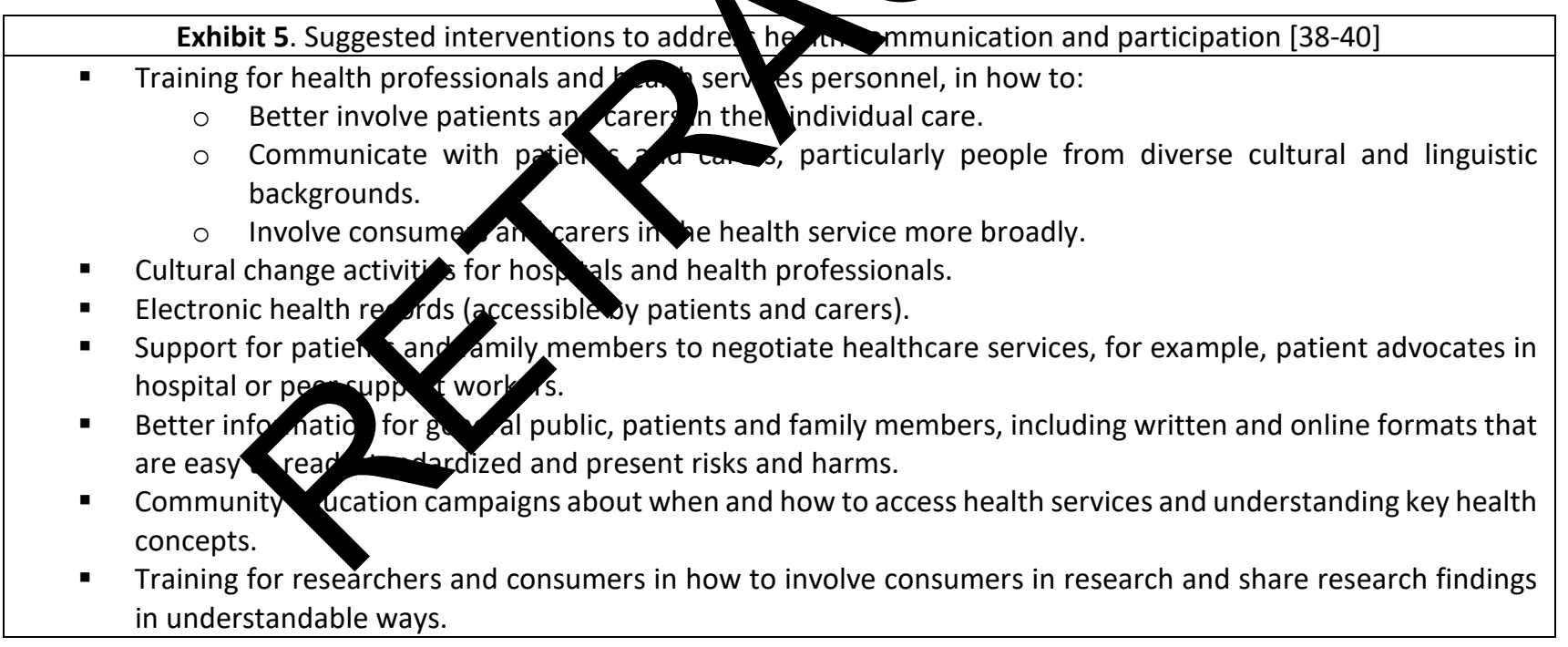

Collaboration with groups of health professionals other than nurses remains relatively unexplored. Due to the large volume of work published on collaboration with nurses, we focused on the other actors within the primary care team. Pharmacistprovided direct patient care has been quantitatively assessed as effective on safety and patient-based outcomes, including medication adherence, patient knowledge and health-related quality of life. In addition, pharmacist-physician quality circles in ambulatory care are cost-effective. Counseling in primary care can be associated with significantly greater clinical effectiveness in short-term mental health outcomes compared with usual care and cause a significant reduction in the number of consultations, prescriptions and referrals to specialist care. It is likely that professionals' beliefs and values are determining factors for collaboration, as it is a complex process beyond the efficacy of some experimentation. Continuity is achieved through the interactions across the patient's circle of care. Communication must be part of the care and the cure. If patients do not have the tools, they need to understand their disease, engage in shared decision making, and manage their health, including following appropriate health guidance, then the intended benefits of medical care and medicines can be 
compromised-or wasted altogether. It is up to all of us in the health community to make improving health literacy part of our calling. It is therefore necessary to better understand actors' perceptions before implementing shared roles and responsibilities between professional groups [39-45].

\section{Conclusion}

The path to safer medication use and improvements in patient safety is not about a destination. This is a journey that must involve iterative learning. There are no absolute solutions, no mystical pronouncements that will tell the profession of pharmacy what to do to fix the system. The problems it faces will not be solved by the level of thinking that created them. The profession is forced to consider new approaches, new knowledge and to consider ways of thinking, acting and being that are outside our traditional approaches. Ultimately, the judge of the quality of work, the services delivered and the outcomes of care is an increasingly well-informed patient, as well as their payors and regulators from the public and private sectors. Focus on patient needs and wants, less on how we do it around here.

\section{Article Highlights:}

1. Around $60 \%$ of medication errors occur during patient admission, discharge or transf of care

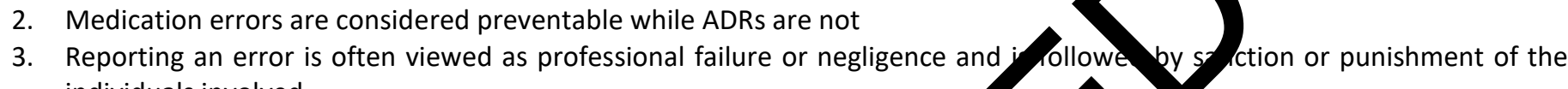
individuals involved.

4. Collaboration is essential to minimize patient risk in the medication use

5. Staff shortage, interruption and SALAD comprises around $30 \%$ errors

6. In 2016, medical errors were estimated to result in 250000 deathann of death in the USA.

7. Medication errors can take place at any stage of choos a medicine, writing the prescription, manufacturing the formulation, dispensing, administration and monitoring ther py.

8. UAE bans handwritten medical prescriptions due to 7,000 dec hs world vide result from illegible handwriting

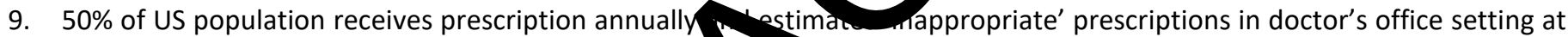
up to $30 \%$.

10. The NHS makes hundreds of millions of prescri arro and mix-ups, leads to 22,300 deaths a year UK, according to a major report commissioned by the Government

11. Unfortunately, in many organizations, production of an error.

12. The IOM estimated costs due to 1 , l errors the US of was approximately $\$ 37.6$ billion/year. About $\$ 17$ billion of it are associated with preventable ersors.

References

1. Rodziewicz TL, Kskind I-Medical Error Prevention. Treasure Island (A. statPearls Publishing; 2018 Jan URL:

https://www.ncbi.nlm.nn.gov/books/NBK499956/

2. Steinwachs DM; Hughes RG. Chapter 8. Health Services Research: Scope and Significance. Patient Safety and Quality: An Evidence-Based Handbook for Nurses. Hughes RG, editor. Rockville (MD): Agency for Healthcare Research and Quality (US); 2008 Apr

3. Smith KE, Enright SM. Providing a Framework for Ensuring Medication Use Safety. Chapter 102. Providing a Framework for Ensuring Medication Use Safety. Remington: The Science And Practice Of Pharmacy. Author: Joseph P Remington; Paul Beringer Publisher: Philadelphia: Lippincott Williams \& Wilkins, (C)2006.
4. Wolf ZR; Hughes GR. Chapter 35Error Reporting and Disclosure. Patient Safety and Quality: An EvidenceBased Handbook for Nurses. Hughes RG, editor. Rockville (MD): Agency for Healthcare Research and Quality (US); 2008 Apr.

5. Pietra LL, Calligaris L, Molendini L, Quattrin R, Brusaferro S. Medical errors and clinical risk management: state of the art. Acta Otorhinolaryngol Ital. 2005 Dec; 25(6): 339-346. PMID: 16749601

6. Nelson EC, Batalden PM, Huber TP, et al. Jt Comm J Qual Improv 2002; 28:472.

7. Kohn LT, Corrigan JM, Donaldson MS, editors. Chapter 8. Creating Safety Systems in Health Care Organizations. To Err is Human: Building a Safer Health System. Institute of Medicine (US) Committee on Quality of Health Care in America. Washington (DC): National Academies Press (US); 2000. 
8. Hughes RG; Blegen MA. Chapter 37Medication Administration Safety. Patient Safety and Quality: An Evidence-Based Handbook for Nurses. Hughes RG, editor. Rockville (MD): Agency for Healthcare Research and Quality (US); 2008 Apr.

9. Thomas JE, Petersen LA. Measuring Errors and Adverse Events in Health Care. J Gen Intern Med. 2003 Jan; 18(1): 61-67. doi: [10.1046/j.15251497.2003.20147.x] PMID: 12534766

10. Reason JT. Human Error. New York: Cambridge University Press; 1990.

11. Ferner RE, Aronson JK. Clarification of terminology in medication errors: definitions and classification. Drug Saf. 2006; 29(11):1011-22.

12. Norman DA. Categorization of action slips. Psychol Rev. 1981;88:1-15.

13. Runciman WB, Sellen A, Webb RK, Williamson JA, Currie M, Morgan C, Russell WJ. The Australian incident monitoring study. Errors, incidents and accidents in anaesthetic practice. Anaesth Intensive Care. 1993;21:506-19

14. Ferner RE, Langford NJ, Anton C, Hutchings A, Bateman DN, Routledge PA. Random and systematic medication errors in routine clinical practice: a multicentre study of infusions, using acetylcysteine as an example. $\mathrm{Br} \mathrm{J}$ Clin Pharmacol. 2001;52:573-7.

15. Weant KA, Bailey AM, Baker SN. Strategies reducing medication errors in the emergench department. Open Access Emerg Med. 2014 doi: [10.2147/OAEM.S64174] PMID: 27147

16. Cheung K, Marcel LB, De Smet AGM errors: the importance of safe disp nsing. J Clin Pharmacol. 2009 Jun; 67(6 76-680. doi: [10.1111/j.1365-2125.2009.03/28.x] PM. : 19594537

17. Pelegrin GM. Medication frors in Hov vitals: An Analysis. Pharmacy Times Octo' 2 r 01, 2004

18. Patel GP, Kane-Gill SL Me ation for analysis: a systematic approar C.Cu Drus ar/2010 Jan;5(1):2-5. PubMed PMID: 2107

19. Morató JF, Ruiz Castell PA, et al Analysis of medication errors in private hospital: pharmacist interventions European Journal of Hospital Pharmacy: Science and Practice 2012;19:102-103.

20. Patel N, Desai M, Shah S, Patel P, Gandhi A. A study of medication errors in a tertiary care hospital. Perspect Clin Res. 2016;7(4):168-173.

21. Cheney C. How to Avoid Medication Errors in Hospitals. Web HealthLeaders October 09, 2018.

22. Wheeler AJ, Scahill S, Hopcroft D, Stapleton $H$. Reducing medication errors at transitions of care is everyone's business. Aust Prescr. 2018;41(3):73-77.
23. Kreckman J, Wasey W, Wise S, Stevens T, Millburg L, Jaeger C. Improving medication reconciliation at hospital admission, discharge and ambulatory care through a transition of care team. BMJ Open Qual. 2018;7(2):e000281. Published 2018 Apr 20. doi:10.1136/bmjoq-2017-000281

24. Wong N. Low Health Literacy: A Concern for Health Insurance Providers and Americans. America's Health Insurance Plans (AHIP) Web October 15, 2018.

25. Sheikh H, Brezar A, Dzwonek A, Yau L, Calder LA. Patient understanding of discharge instructions in the emergency department: do different patients need different approaches?. Int J Emerg Med. 2018;11(1):5. Published 2018 Feb 8. doi:10.1186/s12245-018-01640

26. Okuyama JH Galvao TF, Silva MT. Healthcare Professional's rception f Patient Safety Measured by the $\forall$ spital $>$ vey $\cap$ Patient Safety Culture: A Syster atic Rev and Meta-Analysis. Scinti wrldJoy Mal. 2018;2018:9156301. Ablished 018 19. doi:10.1155/2018/9156301 Co ver J, Whams $\mathrm{H}$, Hibbert $\mathrm{P}$, et al. Classification of patie, safety incidents in primary care. Bull World Health Organ. 2018;96(7):498-505.

28. A well A-M, Burns EM, Hull L, Mayer E, Sevdalis N, rzi A. International recommendations for national patient safety incident reporting systems: an expert Delphi consensus-building process. BMJ Qual Saf. 2017. February;26(2):150-63

29. Scheffler AL. Useful information on the world wide web BMJ Quality \& Safety 2002;11:101-102.

30. Britten N. Medication errors: the role of the patient. $\mathrm{Br}$ J Clin Pharmacol. 2009 Jun; 67(6): 646-650. doi: [10.1111/j.1365-2125.2009.03421.x] PMID: 19594532

31. Shahid S, Thomas S. Situation, Background, Assessment, Recommendation (SBAR) Communication Tool for Handoff in Health Care - A Narrative Review. Safety in Health, 2018, Volume 4, Number 1, Page 1

32. Braaf S, Rixon S, Williams A, Liew D, Manias E. Medication communication during handover interactions in specialty practice settings. J Clin Nurs. 2015 Oct;24(19-20):2859-70. doi: 10.1111/jocn.12894. Epub 2015 Jul 16. PubMed PMID: 26178317.

33. Kitson NA, Price M, Lau FY, Showler G. Developing a medication communication framework across continuums of care using the Circle of Care Modeling approach. BMC Health Serv Res. 2013;13:418. Published 2013 Oct 17. doi:10.1186/1472-6963-13418 
34. Price M. Circle of care modelling: an approach to assist in reasoning about healthcare change using a patientcentric system. BMC Health Serv Res 2016 Dec 04;16(1):546

35. Pandolfe F, Crotty BH, Safran C. Medication Harmony: A Framework to Save Time, Improve Accuracy and Increase Patient Activation. AMIA Annu Symp Proc. 2017;2016:1959-1966. Published 2017 Feb 10.

36. Liu W, Manias E, Gerdtz M. Medication communication through documentation in medical wards: knowledge and power relations. Nurs Inq. 2014 Sep;21(3):246-58. doi: 10.1111/nin.12043. Epub 2013 Jul 4. PubMed PMID: 23822212.

37. Smith KJ, Handler SM, Kapoor WN, Martich GD, Reddy VK, Clark S. Automated Communication Tools and Computer-Based Medication Reconciliation to Decrease Hospital Discharge Medication Errors. Am J Med Qual. 2015;31(4):315-22.

38. Synnot A, Bragge $P$, Lowe $D$, et al. Research priorities in health communication and participation: international survey of consumers and other stakeholders. BMJ Open. 2018;8(5):e019481. Published 2018 May 8. doi:10.1136/bmjopen-2017019481

39. Supper, O. Catala, M. Lustman, C. Chemla, Y. Bourgueil, L. Letrilliart; Interprofessional collaboration in primary health care: a review of facilitators an barriers perceived by involved actors, Journal of Public Health, Volume 37, Issue 4, 1 December 2017ges 716-727, https://doi.org/10.1093/pubmed du10
40. Price M, Lau FY. Provider connectedness and communication patterns: extending continuity of care in the context of the circle of care. BMC Health Serv Res. 2013;13:309. Published 2013 Aug 14. doi:10.1186/1472-6963-13-309

41. Lee JI, Cutugno C, Pickering SP, et al. The patient care circle: a descriptive framework for understanding care transitions. J Hosp Med. 2013;8(11):619-26.

42. McNeil K, Gemmill M, Abells D, et al. Circles of care for people with intellectual and developmental disabilities: Communication, collaboration, and coordination. Can Fam Physician. 2018;64(Suppl 2):S51-S56.

43. Rosenblatt M, Myers L. Communication Is Part of the Cure: Improving Literacy. J Health Commun. 2016;21 Suppl (sup1):1

44. Hunchak C, Ta enbaum, Roberts M, Shah T, Tisma $P$, Ovens C, Borga dvaa 3 . Closing the circle of care: imple entati of a eb-based communication tool to im, en rgency department discharge mmunto rion vith family physicians. CJEM. 2015 Mu 17(2):12 30. doi: 10.2310/8000.2014.141327. Publh d PMID: 25927256.

45. Aguirre-Duarte NA. Increasing collaboration between alth professionals: Clues and challenges. Colomb ed (Cali). 2015;46(2):66-70. Published 2015 Jun 30. 